\title{
The flexible use of inductive and geometric spatial categories
}

\author{
L. Elizabeth Crawford • Erin L. Jones
}

Published online: 5 April 2011

(C) Psychonomic Society, Inc. 2011

\begin{abstract}
The present study examined the flexibility with which people can adopt different category schemes in the spatial domain. In a location memory task, participants viewed and estimated the locations of four kinds of objects that were spatially grouped by object identity. This identitybased arrangement was either congruent or incongruent with the perceptually based, geometric categories that have been reported in previous research. Four experiments examined the conditions under which these different category schemes are used to inform estimates of locations. The results showed that use of identity information depended on the number of objects to be remembered during a trial: When one or two objects were remembered at a time, only geometric categories affected estimates, but when four objects were to be remembered, both geometric categories and identity groupings affected estimates. As memory load increases, participants rely on additional sources to inform their estimates of location.
\end{abstract}

Keywords Categorization · Spatial cognition · Memory

People use multiple categorization schemes to classify items. Which categories we apply in a given situation depends on several factors, including current goals and recent experiences. For example, we may use taxonomic categories to classify an apple as a fruit but, under other

L. E. Crawford ( $\square)$

Department of Psychology, University of Richmond,

Richmond, VA, USA

e-mail: lcrawfor@richmond.edu

E. L. Jones

Department of Psychology, University of Illinois,

Champaign, IL, USA circumstances, may use goal-derived categories to classify it as a snack food (Ross \& Murphy, 1999). The present study examined such category flexibility in the spatial domain.

There is mixed evidence about the flexibility of spatial categorization. One line of research on immediate memory for locations within a spatial frame has shown that people consistently organize space into regions (i.e., categories) that are bounded by the frame's vertical and horizontal axes of symmetry (e.g., Haun, Allen,\& Wedell, 2005; Huttenlocher, Hedges, \& Duncan, 1991; Sampaio \& Wang, 2009, 2010). Furthermore, some experiments have shown that participants continue to use these default quadrant categories even when an alternative categorization scheme would better capture the way stimuli are distributed within the space, suggesting a lack of flexibility (e.g., Huttenlocher, Hedges, Corrigan,\& Crawford, 2004). In contrast, other studies have shown that people derive new spatial categories from recently experienced stimulus distributions (e.g., Hund \& Plumert, 2003, 2005). Although both of these lines of research examined memory for stimulus locations, their procedures differed in ways that may have produced different task demands. Their different conclusions may reflect people's sensitivity to those demands, indicating that people can flexibly adopt alternative spatial categorization schemes as needed.

\section{Geometrically defined spatial categories}

It is well known that the way space is organized into categories influences estimates of stimulus locations (e.g., Friedman, 2009; Huttenlocher et al., 1991; Stevens \& Coupe, 1978). One common finding is that estimates of stimuli presented within a circle are biased away from the circle's horizontal and vertical axes of symmetry and 


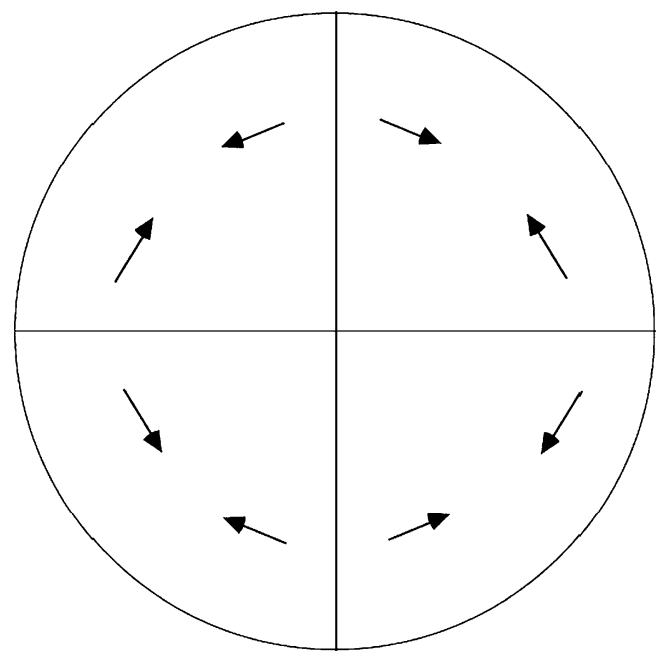

Fig. 1 Schematic memory biases for locations within a circular frame

toward diagonal locations, as in Fig. 1 (Haun et al., 2005; Huttenlocher et al., 2004; Huttenlocher et al., 1991;Wedell, Fitting, \& Allen, 2007). According to the category adjustment model (e.g., Huttenlocher et al., 1991), these biases in estimates of location are a by-product of a Bayesian reconstructive memory process in which inexact memory of a stimulus's particular location is combined with information about the quadrant in which the stimulus appeared. Blending these sources of information causes estimates of location to be assimilated toward quadrant centers (prototypes) and shifted away from quadrant boundaries. Although using these quadrant categories in reconstructive memory introduces bias, Huttenlocher et al. (1991) argued that it can decrease the potential variability in responses, thus making estimates more accurate than they would have been had the category information not been used.

In its original formulation, this model did not address the question of why people consistently carve space into these four quadrant categories, but other work has suggested that this organization is derived from perceptual processes. Across a variety of tasks, horizontal and vertical orientations have been shown to be perceptually privileged. Studies of symmetry detection, spatial discrimination, and memory show that performance is better at these vertical and horizontal orientations than at other orientations (Appelle, 1972; Simmering, Spencer, \& Schöner, 2006; Wenderoth, 1994). The precise coding of these axes is important in both processing-level and computational-level accounts of spatial memory biases. Taking a processing-level approach, dynamic field theory (e.g., Simmering \& Spencer, 2007; Simmering et al., 2006) shows how the cardinal axes of symmetry can serve as reference frames within long-term memory that repel short-term memories of spatial location. Taking a computational-level approach, the category adjustment model (Huttenlocher et al., 2004) suggests that because these axes are coded so precisely, using them as category boundaries enables people to classify locations very accurately, which is important because errors in classification can lead to grossly inaccurate memories.

Such perceptual factors may provide a default organization for an otherwise undifferentiated space, but there are many other possible bases for categorization. Given the ease with which people encode spatial and frequency information about objects (e.g., Hasher \& Zacks, 1979), we might expect that they would use information about the spatial distributions of objects to organize space into categories. For example, if items tend to cluster in certain regions of space, people might categorize that space accordingly, centering categories on those clusters and placing boundaries at regions of low density between the clusters. Given that categories influence memory, there is an obvious advantage to adopting categories that actually reflect the distribution of objects in the world (i.e., that carve nature at its joints). All else being equal, such categories would improve overall memory accuracy by biasing estimates of location toward regions where the stimuli are more likely to have appeared and away from less likely regions. Recent studies have examined the role of inductive experience in spatial categorization, with mixed results.

\section{Inductive experience in spatial categorization}

One approach to studying spatial categorization has been to use a serial reproduction task in which identical items appear in different locations and participants estimate the location of each object immediately after it has been shown. Using this procedure, Huttenlocher et al. (2004) examined the role of inductive experience in spatial categories by presenting dots that were densely clustered around the cardinal axes within a circle, appearing only rarely at diagonal locations. They found no evidence that people were sensitive to this manipulation. People retained the default quadrant categories, biasing estimates away from the cardinal axes, which were high-density regions, and toward diagonals, which were low-density regions. In contrast, other serial reproduction studies have shown that estimates can be influenced by experience with stimulus distributions. For example, using a task similar to Huttenlocher et al.'s (2004), Lipinski, Simmering, Johnson, and Spencer (2010) manipulated stimulus distributions and analyzed bias in estimates over the course of four blocks of trials. Although participants continued to use the default categories throughout the experiment, there was a change in estimates across blocks that depended on the distribution shown, suggesting that the impact of distribution emerges as exposure to it increases. Using a different type of display and more radically different distributions, Spencer and Hund (2002) also found that 
immediate estimates of location were affected by prior experience with stimuli. In addition, Crawford and Duffy (2010) found that estimates of location were influenced by the spatiotemporal distribution of locations that participants had experienced previously. In these studies, people were found to be somewhat sensitive to variations in spatial distribution of stimuli, even though they did not use that information to override the default category structure.

Another approach to examining experience in spatial categorization has been to present a set of unique objects and then have participants reproduce all of the objects in a subsequent block, using the object's identity as a retrieval cue for location. For example, Hund and Plumert (2005) trained people on the locations of 20 objects and manipulated whether the objects were learned in random order or were grouped by both spatial location and temporal order. Location memory was tested in a subsequent block, and the results showed that participants who learned grouped objects used those groupings when reproducing object locations. In addition, Hund and Plumert (2003) and Hund and Foster (2008) taught children and adults the locations of four different kinds of objects, which, in some cases, were spatially grouped by kind (i.e., all of the animals appeared in one region, the vehicles in another, etc.). After studying all of the objects, participants reproduced the location of each one, and their estimates showed that those who had learned the objects in identity clusters used those clusters to relocate individual objects. In addition, Maddox, Rapp, Brion, and Taylor (2008) found that participants integrated information about the race of community members when learning a map of a fictitious community and that this information affected estimates of map locations. These studies suggest that at least under certain blocked testing conditions, people's estimates are influenced by distributional information. Such information is useful because, when objects are grouped in space by identity, an item's identity then provides constraints on the possible range of locations in which that object could have appeared.

Additional work has examined factors that influence the degree to which estimates of location incorporate identity-based spatial groupings. Specifically, Recker and Plumert (2008) studied whether people rely more on such groupings when they are given the opportunity to view all of the objects simultaneously. As in the Plumert and Hund work described above, the objects were spatially grouped by kind, and some participants viewed all 20 objects simultaneously before estimating the individual object locations. However, in another condition, participants viewed each object individually. They viewed all of the studied locations in a single study block before estimating locations, but they never saw the entire distribution at once. While adults' estimates were affected by the category grouping under both viewing conditions, 11-year-old children appeared to use this information only when they had seen all the objects simultaneously (Recker \& Plumert, 2008, Experiment 2). The authors note that viewing objects simultaneously may focus participants' attention on the spatial groups and facilitate processing of configural information, thus increasing the relative strength of the spatial groups in memory.

Combining aspects of the blocked testing and serial reproduction approaches, recent findings suggest that participants sometimes make use of identity-based categories even when immediately estimating the location of a single stimulus. Sampaio and Wang (2010) had participants view a single object within a circle and then estimate its location from memory, as in the serial reproduction studies described above. However, this study differed in that it used four different objects that were spatially grouped by identity, so that each object type appeared only in one region of space, creating identity-based regions within the circle. Before beginning the serial reproduction task, participants were shown an image of the circle containing the entire distribution of objects, making it clear from the start that objects were spatially grouped by identity. These identitybased regions were centered on the circle's horizontal and vertical axes and so were misaligned with the default categories, which are bounded by these axes. Sampaio and Wang (2010) reported that estimates were biased toward the cardinal axes, suggesting that participants adopted a categorization scheme based on object identity distributions, rather than on the default geometric categories.

\section{The present experiments}

In the present study, we sought to reconcile people's failure to use experience-derived categories in some spatial contexts with their adoption of them in others. Assuming a general goal of accurate memory, the benefit of these alternative categorization schemes likely depends on the certainty with which they can be used for classification. Here, we considered how the number of objects participants are asked to remember within a trial may influence the relative certainty of classifications within these two schemes.

In each of four experiments, we used a distribution of four kinds of objects that were spatially clustered by object identity in a manner that was either congruent or incongruent with the default quadrant categories. Participants performed a serial reproduction task in which they viewed a single object or a set of individually shown objects drawn from one of the distributions and then were given each object again and were asked to move it back to its prior location. Unlike in Sampaio and Wang's (2010) study, participants were not shown the object distribution at the 
beginning of the experiment; in order to use it, they had to learn it through trial-by-trial experience. With this approach, we found that they did not use identity-based categories when estimating a single object. ${ }^{1}$ We then showed that increasing the number of objects to be remembered led people to shift from geometric to identity-based categories. Our final experiment directly compared two-object and four-object versions of the task that had comparable timing parameters and showed an effect of stimulus distribution on estimation only in the four-object version. This experiment also included an alternative assessment of distribution learning, the results of which suggested that participants in both twoobject and four-object versions were sensitive to the difference in stimulus distributions, even though those in the two-object version did not appear to use the distribution during estimation.

We suggest that as the number of objects to be remembered increases, this task makes different demands on the identity-based and geometric category schemes. When very few objects are to be remembered, people may rely on geometric categories, because their precise boundaries make costly categorization errors very unlikely (Huttenlocher et al., 2004). When the number of objects to be remembered increases, memory is likely to worsen and lead to more confusion about which object appeared at which location. Under the geometric category scheme, it is the object's remembered location that determines its category membership, and so categorization will become less reliable as memory for location becomes less reliable. In contrast, an object's membership in the identity-based categorization scheme is provided at test by the identity of the object and does not depend on memory for the particular object being tested. Thus, even if memory for the object's particular location is extremely poor, its identity will indicate the region of space in which it appeared, assuming that the identity categories have been learned. If people flexibly adopt categorization schemes to suit the demands of a given situation, they might rely less on geometric categories and more on identity-based categories when trying to remember several objects at once.

\section{Experiment 1: Single stimulus recall}

Method

Participants Twenty-six undergraduates at the University of Richmond participated in exchange for partial course credit.

\footnotetext{
${ }^{1}$ Additional methodological differences that might account for these discrepant findings are considered in the Discussion section of Experiment 1.

Stimuli and procedure After informed consent procedures, each participant was seated at a PC with 43.2-cm (i.e., 17 -in. diagonal) monitor set to a resolution of 38 pixels $/ \mathrm{cm}$. It was explained that they would see an object within a circle and then would reproduce the object's location from memory in a comparable circle. There were four different stimulus objects used in the experiment: a pink diamond, a blue square, a red dot, and a yellow hourglass. Each object was approximately $13 \times 13$ pixels. The objects appeared at the same radial location ( 240 pixels from the circle's center) and at 120 unique angular locations, every $3^{\circ}$ around the circle.

Participants were randomly assigned to view stimuli that were grouped so as to be either congruent or incongruent with the default quadrant organization. The stimulus distributions are shown in Fig. 2. In the congruent condition, the blue squares appeared only between $0^{\circ}$ and $90^{\circ}$, the red dots between $90^{\circ}$ and $180^{\circ}$, the yellow hourglasses between $180^{\circ}$ and $270^{\circ}$, and the pink diamonds between $270^{\circ}$ and $360^{\circ}$ (all ranges are inclusive). Thus, the central location for each object type was at the center of one of the four quadrants $\left(45^{\circ}, 135^{\circ}, 225^{\circ}\right.$, and $\left.315^{\circ}\right)$. In the incongruent condition, the distribution was rotated $45^{\circ}$,so that the blue squares appeared between $45^{\circ}$ and $135^{\circ}$, the red dots appeared between $135^{\circ}$ and $225^{\circ}$, the yellow hourglasses appeared between $225^{\circ}$ and $315^{\circ}$, and the pink diamonds appeared between $315^{\circ}$ and $45^{\circ}$. In this condition, the central location of each object type was on one of the quadrant boundaries $\left(0^{\circ}, 90^{\circ}, 180^{\circ}\right.$, and $\left.270^{\circ}\right)$. Each location was shown once, except for the locations at the object-category boundaries, which were shown twice (once with each possible object), resulting in 124 trials.

On each trial, a circle (668-pixel diameter) was displayed for $2,000 \mathrm{~ms}$, and then one of the four stimuli, drawn at random without replacement, appeared within the circle for $600 \mathrm{~ms}$. The screen went blank $(800 \mathrm{~ms})$, and then the circle reappeared, shifted 100 pixels to the right. $^{2}$ After a pause $(500 \mathrm{~ms})$, the object reappeared in the center of the circle, and participants used the mouse to move it to the location where it had just been shown, relative to the circle. A response was registered when the participant pressed the mouse button.

\section{Results and discussion}

Data from 1 participant were removed because his or her responses had an average absolute error (i.e., absolute

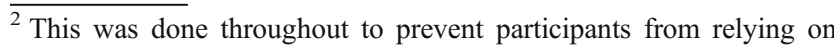
any unplanned landmarks, such as a smudge on the monitor, to aid their memory.
} 

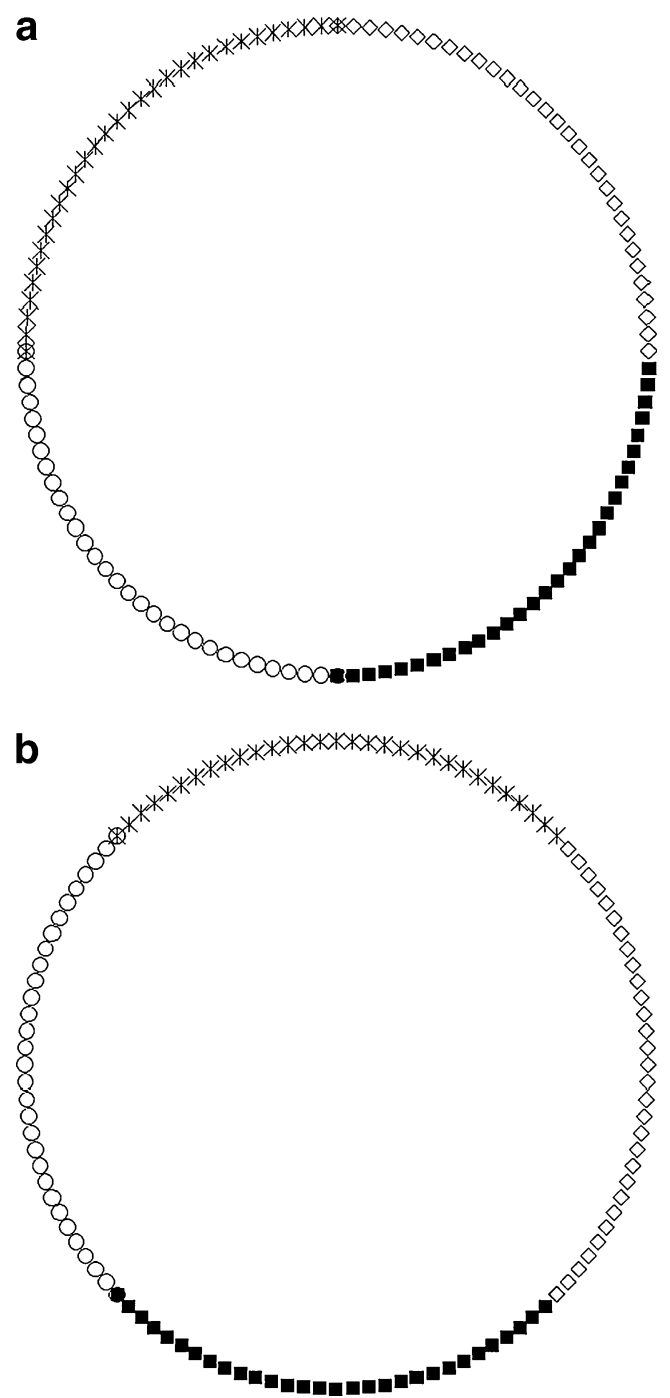

Fig. 2 Congruent (a) and incongruent (b) stimulus distributions

distance between stimulus and estimate) that was more than 3 standard deviations above the mean absolute error by participant. In addition, we removed individual responses that had either an absolute error more than 3 standard deviations above the mean absolute error or an angular error more than 3 standard deviations above the mean angular error. Combined, these culling procedures removed $5.6 \%$ of the estimates.

Angular bias was calculated as the signed difference in degrees between the angular position of each stimulus and the angular position of the estimate of that stimulus and was coded so that the most extreme possible bias was $\pm 180^{\circ}$. Positive values indicated counterclockwise bias, and negative values indicated clockwise bias. Thus, if estimates were biased toward the center of the default quadrant categories without being affected by the distribution manipulation, plotting angular bias against actual angular location would produce a negatively sloping curve within each quadrant for both conditions. Alternatively, if participants used the identity-based categories in estimation, their estimates would be biased toward those categories, either reversing or attenuating this negative slope for the incongruent condition.

Angular bias in estimates was plotted against true angular position in Fig. 3a for the congruent condition and Fig. $3 b$ for the incongruent condition. The data mirrored the within-quadrant negatively sloping bias curves reported in previous work, since estimates were biased away from the quadrant boundaries and toward quadrant centers. To test for a difference between conditions, we collapsed across the four quadrants by coding each stimulus angle relative to its position within the quadrant, thus treating $93^{\circ}, 183^{\circ}$, and $273^{\circ}$ as comparable to $3^{\circ}$. Average angular biases at each angle (except $0^{\circ}$, the default boundary) were regressed on true angular position, including a dummy variable for condition in order to test for differences in the slope between the two conditions. There was no significant difference [congruent condition slope $=-.068$, incongruent condition slope $=-.074$, slope difference $=.006$; $t(54)=1.24$, n.s.]. Each of these slopes was significantly
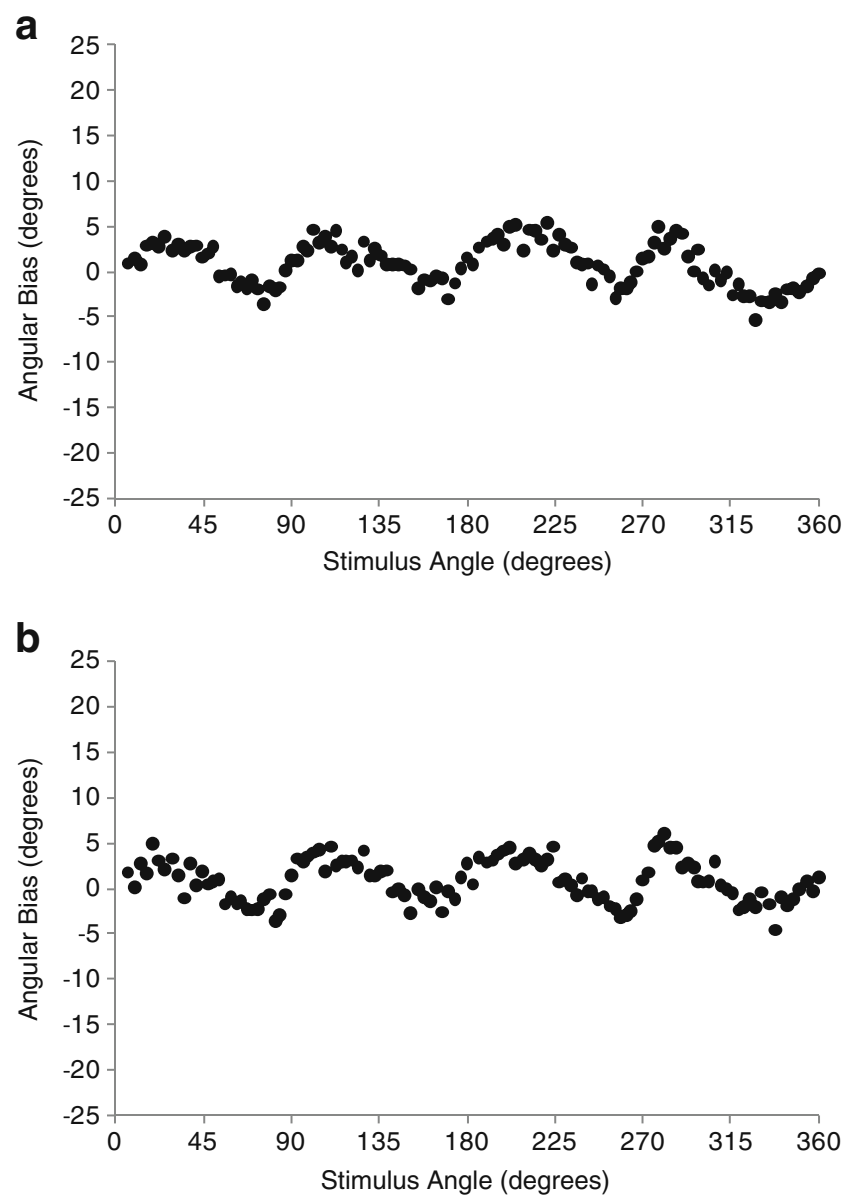

Fig. 3 Experiment 1: Bias by stimulus angle for congruent (a) and incongruent (b) conditions 
different from zero [congruent condition, $t(27)=-8.76$, $p<.001$; incongruent condition, $t(27)=-10.90, p<.001$ ] In addition, an independent samples $t$-test on the mean absolute error by participant revealed no significant difference in error between the two conditions [congruent condition $=21.88$ pixels, incongruent condition $=20.82$ pixels; $t(23)=-0.685$, n.s.].

If the identity-based categories are learned over time, we might expect their effects on bias to emerge only in later trials, as in Lipinski et al. (2010). We divided the trials into three temporal segments (early, middle, and late trials) and examined the slope of the bias curves by segment. There were no significant main effects or interactions involving the segment variable in this or in any of the experiments that follow. It should be noted that this experiment used fewer trials than in Lipinski et al. and that an impact of identity-based categories may emerge only after participants gain more experience with object distributions.

The results indicate that participants used the default quadrant categories to estimate location but provide no evidence that they used identity clusters.It is possible that participants did not learn the identity-based categories well enough to use them in estimation. Such an interpretation might be suggested by the discrepancy between these results and those in Sampaio \& Wang (2010), which did show an effect of identity clusters on single-object estimations. In that study, participants were shown the entire distribution of objects at the start of the experiment and were told explicitly that stimuli were divided into categories that corresponded to object identities. In contrast, the present study used an incidental learning approach, examining whether people would learn and apply the object organization spontaneously. This approach, which has been widely used in studies of inductive categorization and statistical learning (e.g., Huttenlocher et al., 2004; Lipinski et al., 2010; Spencer \& Hund, 2002) may have made learning the inductive categories more difficult than in Sampaio and Wang's (2010) study. It is also possible that participants ignored identity information because it seemed irrelevant to the task, which asked only for the last-seen location.

\section{Experiment 2: Recalling two stimuli}

When asked to view and estimate the location of one object at a time, participants appear to have ignored potentially useful information about the identity of objects. However, previous work suggests that participants do make use of identity-based categories in estimation when reproducing locations of several objects (Hund \& Foster, 2008; Hund \& Plumert, 2003). The number of objects to be remembered may determine whether or not people make use of identity- based categories. When more than one object is to be estimated within a trial, participants must use the identity of the object as a retrieval cue for the location of that object. This cued-recall task requires the integration of identity and location information. Experiment 2 investigated whether adding an additional object and, thus,requiring identity and location integration would lead participants to use identitybased information to inform their estimates, thus attenuating the negatively sloping bias when the identity-based categories were incongruent with the geometric categories.

\section{Method}

Participants Thirty-seven University of Richmond undergraduates participated in exchange for partial course credit.

Stimuli and procedure The materials, procedure, and timing parameters were the same as those in Experiment 1, except that after the first stimulus object was shown within the circle and then removed, there was an 800 -ms pause before another stimulus was presented for $600 \mathrm{~ms}$. This second object was randomly chosen from the distribution, with the constraint that it had to have a different identity from the one just shown. Once the second object was removed, the circle was shifted rightward, and then one of the two studied objects appeared in the center of the circle and participants moved it to its prior location. The object then disappeared, and the other studied object appeared in the center of the circle and was relocated. The order of the two response objects was random, and thus it did not necessarily correspond to the order in which the objects were shown for study. This randomization was important because it obviated using the temporal order of stimuli to do the task; thus, it was emphasized during the instructions. There were 72 estimation trials. It should be noted that increasing the number of stimuli from one to two also increased the average delay between stimulus and response. We addressed the issue of timing parameters directly in Experiment 4.

\section{Results and discussion}

The same culling criteria were applied as in Experiment 1, eliminating $4.2 \%$ of the estimates. Bias was calculated and angular positions were collapsed across the four quadrants as in Experiment 1. The average bias by angular position for the congruent and incongruent conditions is presented in Fig. $4 \mathrm{a}$ and $\mathrm{b}$. The results showed that in both conditions, estimates were biased away from the major axes and toward diagonal locations. The regression slopes for the two conditions were not significantly different from each other [slope difference $=-.002 ; t(54)=-0.113$, n.s.]. In both conditions, the slope was negative and significantly 

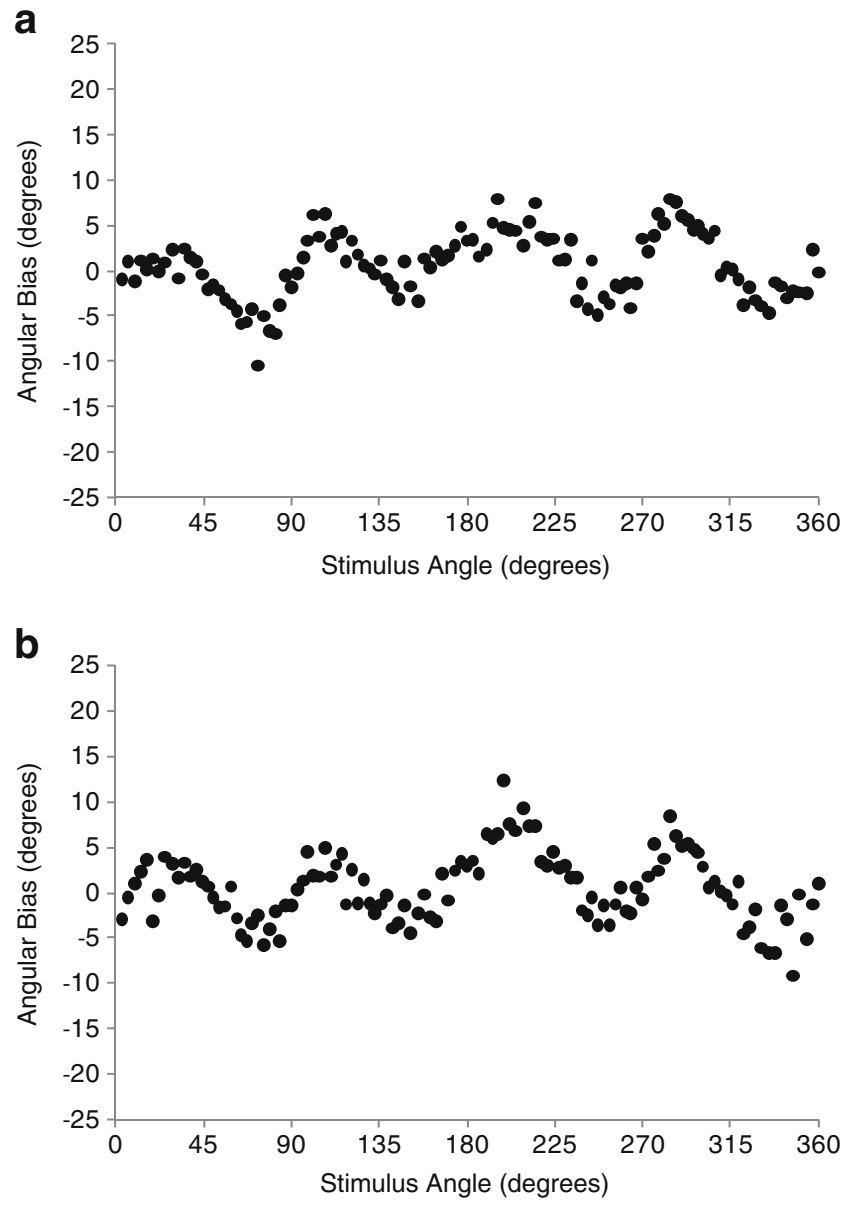

Fig. 4 Experiment 2: Bias by stimulus angle for congruent (a) and incongruent (b) conditions

different from zero [congruent condition slope $=-.094$, $t(27)=-7.16, p<.001$; incongruent condition slope $=-.092$, $t(27)=-6.84, p<.001]$. In addition, there was no difference in absolute error between the two conditions [congruent condition $=33.85$ pixels, incongruent condition $=35.01$ pixels; $t(35)=0.563$, n.s.].

This task asked participants to estimate two locations, requiring that they remember which object went with each location. Thus, it required that identity and spatial information be bound in memory. In spite of this, there was no evidence that participants formed categories that captured the distribution of object identities or used those categories to estimate locations. Whether the object groupings were aligned or misaligned with the default geometric categories, estimates were affected by the quadrant categories only.

\section{Experiment 3: Recalling four stimuli}

Above, we noted two reasons why people might take advantage of identity-based categories when remembering multiple objects. One, multiple objects require people to integrate spatial and location information; yet Experiment 2 required this and still showed no use of identity-based information in spatial memory. Two, increasing the number of objects changes the relative benefit of using identity-based and geometric categories. We next examined memory for four objects, which is about the capacity limitation shown in other visual working memory research (Cowan, 2000).

Method

Participants Thirty-two University of Richmond undergraduates participated in exchange for partial course credit.

Stimuli and procedure The experiment used the same materials and manipulation as in the previous two but presented four objects to be remembered within a trial. Stimulus presentation times were lengthened in order to reduce the difficulty of the task. On each trial, the circle appeared for $2,000 \mathrm{~ms}$, and then one randomly selected object appeared within it for $800 \mathrm{~ms}$. Once the object was removed, there was a $1,000-\mathrm{ms}$ pause, and then the next object appeared for $800 \mathrm{~ms}$. Each object was randomly selected, with the constraint that it had to be different from the objects already presented within the trial; thus, the four objects shown had different identities. This process was repeated for each of four objects. Once the fourth object was removed, there was another 100-ms pause, and then the circle shifted 100 pixels to the right. After $500 \mathrm{~ms}$, one of the four studied objects appeared in the center of the circle, and participants moved it to its prior location within the circle. This was repeated four times, with the object erased once the response had been made. At the response stage, the order of the four objects was random, and this was emphasized to the participants. There were 3 practice trials, followed by 42 trials.

\section{Results and discussion}

Applying the same criteria as in the previous experiments, $4.4 \%$ of the estimates were removed.

The average bias by angular position for the congruent and incongruent conditions is presented in Fig. 5a and b. The graphs make clear that the previously observed sinusoidal pattern with negative bias slopes for each quadrant was replicated in the quadrant congruent condition, but not in the incongruent condition. As in Experiment 1, we collapsed angular locations across quadrants and ran a regression of bias on actual stimulus angle and condition. There was a significant difference in the slope of the regression line between the two conditions [congruent condition slope $=-.18$, incongruent condition slope $=+.02$, slope difference $=.20 ; t(54)=6.59, p<.001]$. Whereas the 

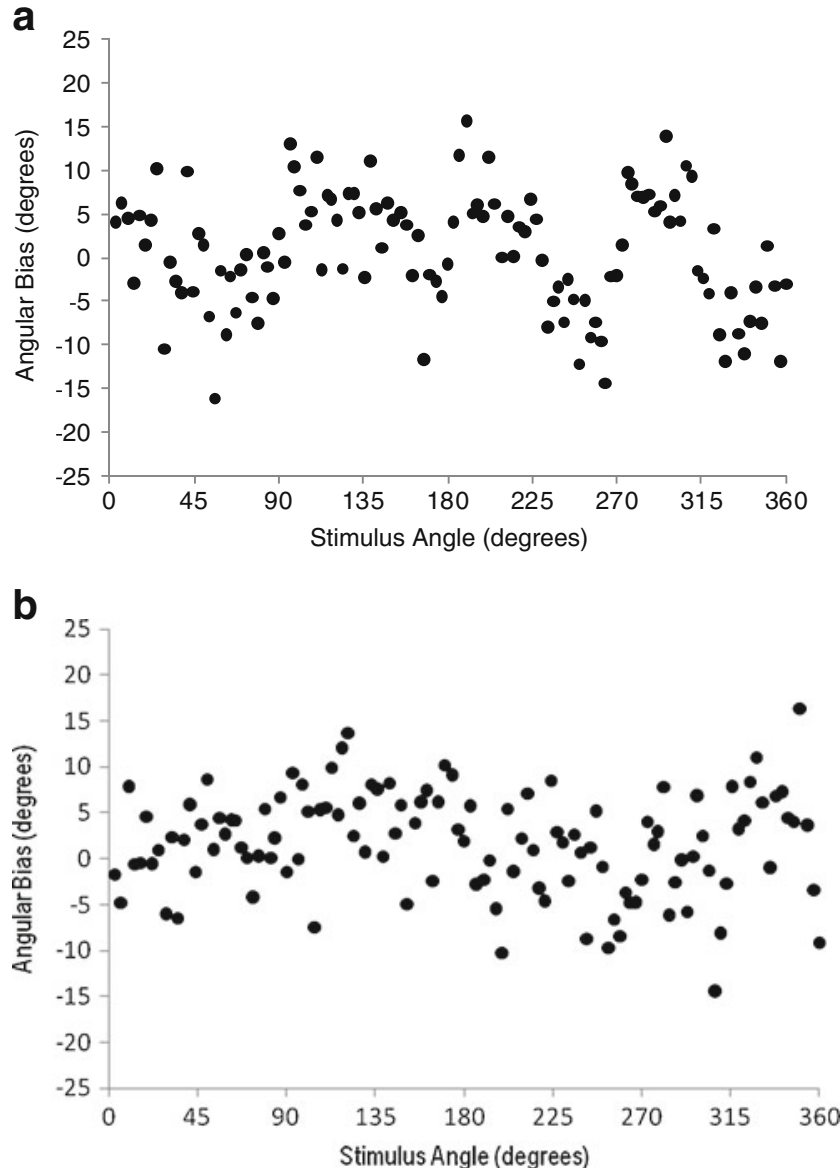

Fig. 5 Experiment 3: Bias by stimulus angle for congruent (a) and incongruent (b) conditions

slope in the congruent condition was negative and significantly different from zero, $t(27)=8.77, p<.001$, the slope in the incongruent condition was not, $t(27)=0.67$, n.s. There was no significant difference in absolute error between the two conditions [congruent condition $=70.05$ pixels, incongruent condition $=61.23$ pixels; $t(30)=-1.03$, n.s.].

The bias analysis presented above does not distinguish the possibility that participants in the incongruent condition combined both category schemes from the possibility that each used only one scheme but individuals chose different schemes. To address this, we examined the bias slopes for each participant. If participants in the incongruent condition used one scheme or the other, some slopes should be negative and some should be positive, but they should be as extreme as those in the congruent condition. In contrast, if individual participants adopted both schemes, the participant-level slopes should be flatter. An independent samples $t$-test on the absolute value of slope estimates for each participant showed that the slopes in the incongruent condition were significantly flatter than those in the congruent condition [incongruent $M=.08$ congruent $M=.16 ; t(30)=2.59, p<.05]$. This suggests that individual participants combined information from both categorization schemes, rather than adopting one scheme or the other.

The results of Experiment 3 show that when participants are asked to remember the locations of four objects during a trial, their estimates are influenced by the identity-based organization of stimuli. When stimulus clusters are incongruent with this default organization, estimates reflect neither the default categories nor the categories suggested by the identitybased clusters. Responses appear to be a compromise between the two organizations. Under these conditions, knowledge that objects of a given identity appear in certain regions of space affects memories of object locations.

\section{Experiment 4: Two versus four objects}

We suggested that increasing the number of objects to remember changes the relative benefit of using geometric versus identity-based categories in stimulus estimation. Participants' use of identity information when remembering four (but not one or two) objects is consistent with the view that they apply these categorization schemes in a flexible way. However, remembering four objects differs from remembering one or two in several ways that may influence reliance on identity-based categories. First, viewing and then reproducing four objects requires a longer delay between presentation and response, and such delays may affect the degree to which people rely on either set of categories (Huttenlocher et al., 1991; Schutte \& Spencer, 2002). Furthermore, our four-object experiment used slower timing parameters. Pilot work had suggested that this was desirable given the difficulty of the task, but it may have changed reliance on the identity-based categories by giving people more time to encode each object's identity and by increasing the delay between presentation and response. In Experiment 4, we examined memory for two versus four objects, while keeping timing parameters comparable.

Experiment 4 also included an additional measure to inform interpretation of the estimation results. Although we have posited that people flexibly apply different categorization schemes, it may be the case that sometimes people simply do not detect the spatial pattern of the presented objects. If people never learn the identitybased categories in the first place, the fact that these categories go unused tells us little about the flexible use of different categorization schemes. In contrast, if people ignore identity clusters in estimation but then use that information on another task, this would indicate that they are applying the identity-based information selectively. For this reason, we added a follow-up task in which participants were asked to indicate the region in which each of the four object kinds appeared. 


\section{Method}

Participants Thirty-eight University of Richmond undergraduates participated in exchange for partial course credit.

Stimuli and procedure The materials were the same as those in the previous experiments. The experiment was a $2 \times 2$ design with distribution (congruent vs. incongruent) and task (four vs. two objects) as between-subjects variables. In the four-object version, participants were shown four objects (one of each object type) to remember and were told that they would be tested on only two of them, whereas in the two-object version, participants were shown two objects and were told that they would be tested on both. In the four-object version of the task, the circle appeared for 2,000 ms, and then each of the four stimuli appeared for $600 \mathrm{~ms}$ within the circle, in random order, with an 800 -ms interstimulus interval. In the two-object version, we used the same procedure, except that we replaced two of the objects with invisible filler objects. Thus, participants in the two-object version viewed an empty circle during the time that those in the four-object version would have been viewing additional objects, making the time between presentation and response comparable across conditions. In both versions, after all of the objects had been shown, the circle was shifted to the right, and then only two objects were reproduced using the same reproduction procedure as in Experiment 2. In the four-object version, these two were chosen randomly from those presented. In the two-object version, these were the only two objects that had been presented.

After completing 72 estimation trials, participants did four retrospective judgment trials. They were told that the items they had just estimated had been arranged so that all appeared at the same radius, forming a ring of stimulus locations within the circle. They were further told that the objects were located "so that each type of object appeared in only one section of the ring and could not appear outside of that section. For example, the red dots could appear at various locations within a certain part of the ring and could not appear anywhere else, the blue squares could only appear in a different section, and so forth." Then the circle was shown, and one of the objects appeared within it. Participants were asked to "think carefully about the region where objects of that type appeared, and then use the mouse to move the object to the center of that region." They made this judgment once for each of the four objects.

Results and discussion

Applying the same criteria as those in the previous experiments, $5.1 \%$ of the estimates were removed. As before, angular bias was collapsed across the four quadrants by coding each stimulus relative to its position within the quadrant. The regression analysis revealed a significant interaction, such that the effect of distribution on regression slope depended on the number of objects shown, $\beta=.24$, $t(108)=6.16, p<.001$. As is shown in Fig. 6a and b, when people remembered only two objects, their estimates replicated the pattern of bias shown in our first two experiments. That is, estimates were biased away from the horizontal and vertical axes of symmetry and toward diagonal locations, regardless of stimulus distribution, since there was no difference in slope between the congruent and incongruent conditions among those who viewed only two objects [congruent condition slope $=-.08$, incongruent condition slope $=-.11$, slope difference $=.03, t(54)=1.09$, n.s.]. Each of these slopes was negative and significantly different from zero [congruent, $t(27)=-4.73, p<.05$; incongruent, $t(27)=-6.22, p<.05]$. However a different pattern emerged for participants who were asked to hold four objects in memory (Fig. 6c and d). Under these conditions, estimates appear to have been sensitive to the way those objects were distributed. When the object grouping aligned with the geometrically defined quadrant categories, there was a strong bias toward the center of those quadrants; however, when the object groupings were misaligned, this bias was eliminated [among those who viewed four objects;congruent condition slope $=-.22$, incongruent condition slope $=-.01$, slope difference $=-.21 ; t(54)=-7.20, p<.001]$. The congruent condition slope was significantly different from zero, $t(27)=-10.95, p<.05$, but the incongruent condition slope was not, $t(27)=-0.54$, n.s.. In addition, among those in the congruent condition, the bias slope was significantly steeper for those asked to remember four objects than for those asked to remember two, $\beta=-.14, t(54)=-5.11$, $p<.001$, whereas the opposite pattern held among those in the incongruent condition, $\beta=.10, t(54)=3.62, p<.001$. This suggests that for both congruent and incongruent distributions, people gave relatively more weight to identity-based categories when remembering four rather than two objects. As in Experiment 3, an independent samples $t$-test on the absolute value of slope estimates for each participant in the four-object incongruent condition showed that these slopes were significantly flatter than those in the four-object congruent condition [incongruent $M=.058$, congruent $M=.208 ; t(15)=7.62, p<.001]$, suggesting that individual participants combined information from both categorization schemes. An analysis of participant-level error showed a main effect of number of stimuli to be remembered, $F(1,34)=6.15, p<.05$, since those remembering four objects were less accurate than those remembering two, but no main effect of distribution and no interaction.

We proposed that having to remember more objects within a trial shifts the relative advantages of relying on 

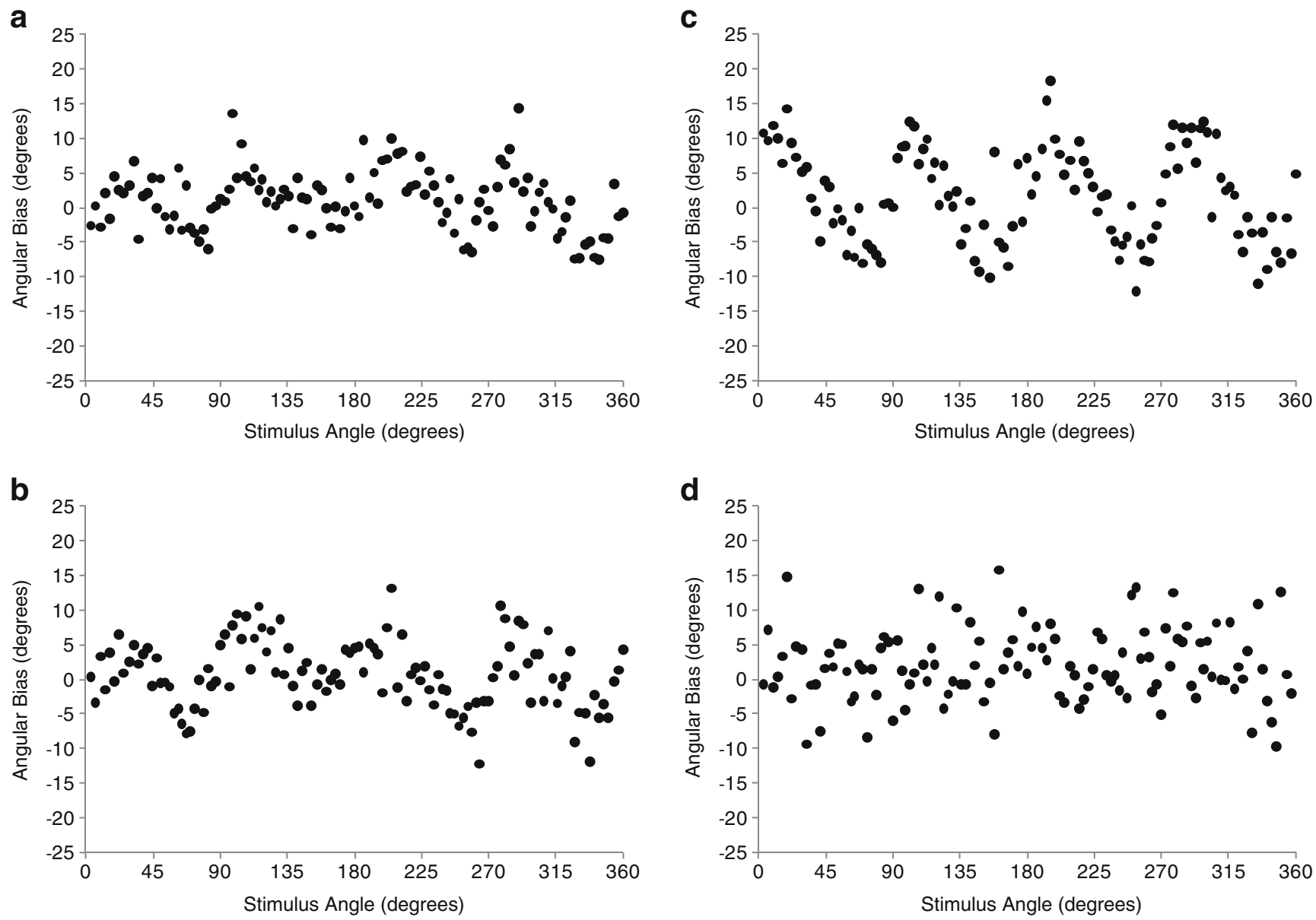

Fig. 6 Experiment 4: Bias by stimulus angle for two-object congruent (a) and incongruent (b) conditions and for four-object congruent (c) and incongruent (d) conditions

identity-based, as opposed to geometric, categories. However, it is also possible that those in the four-object version of the task used the identity information because they had more exposure to it. On any given trial in the experiment, although participants in the two- and fourobject versions had estimated the same number of stimuli, those in four-object conditions had seen twice as many stimuli. Their sensitivity to identity-based categories may have emerged during the second half of trials, when exposure to stimuli had surpassed that of the participants in the twoobject conditions. If so, then among participants who viewed four objects, we should have seen greater impact of the identity-based categories during the second half than during the first half of the trials. Instead, we found that the pattern of bias was indistinguishable between the first and second halves of the trials. Adding half as a dummy variable to the regression analysis of the four-object conditions revealed no significant interaction between trial half and distribution, $\beta=.025, t(108)=.506$, n.s.. Furthermore, the impact of the stimulus distribution within the four-object version was well established during the first half of trials, showing a significant effect of distribution during the first half alone, $\beta=.7 .92, t(54)=4.36, p<.001$, a difference that was not found when all of the data from the two-object version were analyzed. Thus, the identity-based category scheme that produced the pattern of bias shown in Fig. 6c and d was clearly well established within the first half of trials, when exposure to the stimulus distribution was comparable to what those in the two-object version experienced later in the experiment.

The retrospective judgments further inform the role of geometric and object-based categories in estimation, allowing us to determine whether participants detected the difference between the distributions, regardless of whether they used it in estimation. Judgments of each object location were submitted to a repeatedmeasures ANOVA with object (i.e., red dot, blue square, etc.) as a within-subjects factor and distribution and number of objects studied as between-subjects factors. The results produced a significant interaction between object type and distribution, $F(3,99)=91.60, p<.001$, indicating that where participants located these objects depended on which distribution they had seen previously. For the congruent 
condition, in which the prototypes were at $45^{\circ}, 135^{\circ}, 225^{\circ}$, and $315^{\circ}$, the mean estimates were $39.02^{\circ}(S E=10.64)$, $122.75^{\circ}(8.52), 230.90^{\circ}(7.18)$, and $331.70^{\circ}$ (20.45). For the incongruent condition, in which the prototypes were $0^{\circ}, 90^{\circ}$, $180^{\circ}$, and $270^{\circ}$, the mean estimates were $25.50^{\circ}$ (22.42), 93.15 $(11.66), 173.04^{\circ}$ (9.34), and $277.04^{\circ}$ (7.87). The three-way interaction between object type, distribution, and number of objects was not significant, $F<1$, suggesting that the effect of distribution on these judgments did not depend on the number of objects the participants tried to remember. Thus, even though those in the two-object condition did not use identity-based information to estimate locations of just-seen stimuli, the retrospective judgments reveal that they were sensitive to the difference between the two distributions. Participants may have made their retrospective judgments by retrieving some exemplars from memory and then using those to infer the relevant regions. Although the same strategy could have been used during the estimation task, apparently it was not. The results suggest that people apply different categorization schemes under different task conditions and are consistent with the notion that spatial categories can be used flexibly.

To examine the accuracy of these judgments, we analyzed participants' mean unsigned angular error, with distribution and number of objects as between-subjects factors. This analysis showed greater error in the incongruent than in the congruent condition, $F(1,33)=5.365, p<.05$; $M_{\text {incongruent }}=38.05$ (6.06), $M_{\text {congruent }}=19.05$ (5.53). There was no evidence that the number of objects to be remembered affected judgment accuracy, $F(1,33)=1.76$, n.s., or interacted with distribution, $F(1,33)=0.05$, n.s. Participants were able to use the identity-based groupings when making retrospective judgments about object locations, but they did so more accurately when those groupings were aligned with the default quadrant categories than when they were misaligned.

\section{General discussion}

Four experiments examined how categories derived from induction and those derived from perception affect memories of object locations within a circle. Objects were distributed so that each region of space was devoted to only one kind of object. These identity-based regions were either aligned or misaligned with the default quadrant categories. When remembering only one or two object locations at a time, participants' estimates of location were influenced only by the default categories, and not the identity-based categories. In contrast, when four objects were to be remembered, participants' estimates of location were affected by both the default quadrant categories and the presented distribution of objects.
Why do people seem to ignore distributional information when remembering very few items? When objects are spatially grouped by identity, the identity of a stimulus provides information about its location, constraining the range of possible locations in which it could have appeared, and yet the present experiments showed no evidence that this information affects estimates when only one or two objects are to be remembered. We suggest that both identitybased categories and geometric categories can provide some benefit to memory accuracyand that the relative benefit of these two schemes changes with the number of objects to be remembered. It may become worthwhile to take advantage of an inductively based categorization scheme only under conditions that make the perceptually available geometric categories more error prone. This seems likely to occur when several objects must be remembered at once.

Estimation tasks such as those used here do not cleanly distinguish the question of how well people learn identitybased categories from the question of how much weight they give these categories during estimation. Even though the objects are viewed sequentially, presenting several to be remembered within a trial may encourage people to code locations relationally and to direct more attention to object distributions. That is, increasing the number of objects may support the same kinds of processing that simultaneous viewing does (Recker \& Plumert, 2008), perhaps because people visualize all four objects at once. This may allow participants to learn the identity-based categories with more certainty than when only a few objects are presented. As a result, people may use identity-based categories when estimating more objects because, in such cases, the identity-based categories are better learned, and not because the geometric categories are more error prone. These possibilities are not mutually exclusive.

The present study addressed the distinction between learning and applying identity-based categories by including an additional task in Experiment 4 that asked participants to locate the identity-based category prototypes. The results show an effect of distribution that did not depend on the number of objects to be learned, suggesting that those in the two-object condition and those in the four-object condition learned their distributions comparably well, at least by this alternative measure. However it should be noted that this task and the estimation task may tap into different representations of the object distribution. A future direction for this research will be to examine more fully how inductive spatial categories are learned and applied not only in estimation, but also in a variety of tasks. As Markman and Ross (2003) noted, categories serve multiple functions, and the representations formed under one set of learning conditions may not serve all category functions.

This study extends earlier work on the flexibility of spatial categories. Research by Huttenlocher et al. (2004) 
characterized the default quadrant categories as fairly rigid, because these categories were not revised to capture regions of high stimulus density. However, other work has shown that inductive information is sometimes used to inform estimates of location (e.g., Hund \& Foster, 2008; Hund \& Plumert, 2003, 2005; Spencer \& Hund, 2002). Recent studies have begun to examine directly what factors lead people to take advantage of distributional information in estimation. This work has suggested that people are more likely to use inductive information when they have more experience with the stimulus distribution (Lipinski et al., 2010), when there are longer delays between stimulus and response (Schutte \& Spencer, 2002), and when they have the opportunity to view all objects simultaneously (Recker $\&$ Plumert, 2008). The work presented here suggests that the number of objects to be remembered may be another factor influencing whether or not people will apply a categorization scheme derived from experience with a stimulus distribution.

The present study highlights a way in which identitybased categories operate differently than quadrant categories in a location memory task. At the time of response, the experiment provides no information about which quadrant an object appeared in. For that information to be used in reconstruction, it must be available in memory. In contrast, the experiment does provide information about which identity-based category an object belonged to. Because the response object has the same identity as the one to be remembered, it signals its membership in an identity-based category. Even if people cannot remember an object's spatial position at all, if they have stored information about where objects of that kind appeared, the identity of the response object provides access to an identity-based spatial region. This category information can be used to inform their estimates of particular locations. As memory load increases and immediate memory for spatial position becomes less exact, identity information should become increasingly valuable.

This study establishes that participants use identity-based information in certain cases, but it is not known exactly how they integrate various sources of information when remembering locations. It may be the case that identity information is not itself blended with location memories but, rather, is used to determine the weighting of the geometric categories. That is, when identity and geometry misalign, participants may cease using geometric categories, thus producing the flat slope we observed when four objects were remembered and the identity clusters were misaligned with the quadrant categories. This seems unlikely to us in light of the ubiquity of categorization behavior. We suggest, instead, that the flat slopes reflect a blending of both geometric and identity-based categories and the biases that might result from the categorization schemes' canceling each other out. This suggests that if the number of items held in memory were increased further, identity-based categories would eventually override geometric ones, leading to a reversal in the pattern of bias when these categories are incongruent.

The combined use of spatial category schemes mirrors the distinction between context-independent and contextdependent categories that Barsalou (1982) applied to object categorization schemes. Context independence implies that the category will always be activated, even when other categorization schemes are also activated. Here, the perceptually based categories may be context independent, activated regardless of task demands or context, while the identity-based categories appear to be context dependent.

Work on object categorization has shown that people flexibly adopt different categorization schemes to meet the demands of a given situation (e.g., Barsalou, 1983; Ross \& Murphy, 1999). Such flexibility has not been addressed widely in work on spatial categorization. The present experiments suggest that even in the restricted situation of recalling the location of a stimulus within a circle, people adapt their category schemes to meet differing situational demands.

Acknowledgements This project was supported by grants from the University of Richmond Arts and Sciences Faculty Research Committee and Undergraduate Research Committee. We thank Stephanie Cohn, Shannon Henry, and Jocelyn Mabie for help with data collection. We thank three anonymous reviewers for their helpful comments on an earlier draft.

\section{References}

Appelle, S. (1972). Perception and discrimination as a function of stimulus orientation: The "oblique effect" in man and animals. Psychological Bulletin, 78, 266-278.

Barsalou, L. W. (1982). Context-independent and context-dependent information in concepts. Memory \& Cognition, 10, 82-93.

Barsalou, L. W. (1983). Ad hoc categories. Memory \& Cognition, 11, 211-227.

Cowan, N. (2000). The magical number 4 in short-term memory: A reconsideration of mental storage capacity. The Behavioral and Brain Sciences, 24, 87-185.

Crawford, L. E., \& Duffy, S. (2010). Sequence effects in estimating spatial location. Psychonomic Bulletin \& Review, 17, 725-730.

Friedman, A. (2009). The role of categories and spatial cuing in global-scale location estimates. Journal of Experimental Psychology. Learning, Memory, and Cognition, 35, 94-112.

Hasher, L., \& Zacks, R. T. (1979). Automatic and effortful processes in memory. Journal of Experimental Psychology: General, 108, 356-388.

Haun, D. B., Allen, G. L., \& Wedell, D. H. (2005). Bias in spatial memory: A categorical endorsement. Acta Psychologica, 118, 149-170.

Hund, A. M., \& Foster, E. K. (2008). Understanding developmental changes in the stability and flexibility of spatial categories based on object relatedness. Developmental Psychology, 44, 218-232.

Hund, A. M., \& Plumert, J. M. (2003). Does information about what things are influence children's memory for where things are? Developmental Psychology, 39, 939-948. 
Hund, A. M., \& Plumert, J. M. (2005). The stability and flexibility of spatial categories. Cognitive Psychology, 50, 1-44.

Huttenlocher, J., Hedges, L. V., Corrigan, B., \& Crawford, L. E. (2004). Spatial categories and the estimation of location. Cognition, 93, 75-97.

Huttenlocher, J., Hedges, L. V., \& Duncan, S. (1991). Categories and particulars: Prototype effects in estimating spatial location. Psychological Review, 98, 352-376.

Lipinski, J., Simmering, V. R., Johnson, J. S., \& Spencer, J. P. (2010). The role of experience in location estimation: Target distributions shift location memory biases. Cognition, 115, 147-153.

Maddox, K. B., Rapp, D. N., Brion, S., \& Taylor, H. A. (2008). Social influences on spatial memory. Memory \& Cognition, 36, 479-494.

Markman, A. B., \& Ross, B. H. (2003). Category use and category learning. Psychological Bulletin, 129, 592-613.

Recker, K. M., \& Plumert, J. M. (2008). How do opportunities to view objects together in time influence children's memory for location? Journal of Cognition \& Development, 9, $434-460$

Ross, B. H., \& Murphy, G. L. (1999). Food for thought: Cross classification and category organization in a complex real-world domain. Cognitive Psychology, 38, 495-553.

Sampaio, C., \& Wang, R. F. (2009). Category-based errors and the accessibility of unbiased spatial memories: A retrieval model.
Journal of Experimental Psychology. Learning, Memory, and Cognition, 35, 1331-1337.

Sampaio, C., \& Wang, R. F. (2010). Overcoming default categorical bias in spatial memory. Memory \& Cognition, 38, 1041-1048.

Schutte, A. R., \& Spencer, J. P. (2002). Generalizing the dynamic field theory of the A-not-B error beyond infancy: Three-year-olds' delay- and experience-dependent location memory biases. Child Development, 73, 377-404.

Simmering, V. R., \& Spencer, J. P. (2007). Carving up space at imaginary joints: Can people mentally impose arbitrary spatial category boundaries? Journal of Experimental Psychology. General, 33, 871-894.

Simmering, V. R., Spencer, J. P., \& Schöner, G. (2006). Reference-related inhibition produces enhanced position discrimination and fast repulsion near axes of symmetry. Perception \&Psychophysics, 68 , $1027-1046$.

Spencer, J. P., \& Hund, A. M. (2002). Prototypes and particulars: Geometric and experience dependent spatial categories. Journal of ExperimentalPsychology: General, 131, 16-37.

Stevens, A., \& Coupe, P. (1978). Distortions in judged spatial relations. Cognitive Psychology, 10, 422-437.

Wedell, D. H., Fitting, S., \& Allen, G. L. (2007). Shape effects on memory for location. Psychonomic Bulletin \& Review, 14, 681-686.

Wenderoth, P. (1994). The salience of vertical symmetry. Perception, 23, 221-236. 